\title{
Some research advances of immune mechanism during infection in China
}

\author{
YANG Wei \\ Institute of Biophyisics, Chinese Academy of Sciences, Beijing 100001, China
}

Received October 15, 2011; accepted October 30, 2011

Citation: Yang W. Some research advances of immune mechanism during infection in China. Sci China Life Sci, 2011, 54: 1153-1155, doi: 10.1007/s11427-011-4258-7

Newly emerging and reemerging infectious diseases continue to pose a substantial threat throughout the world. Understanding the immune mechanism during infection contributes not only to discoveries in science but also to advances in health care. Chinese researchers have made tremendous efforts to decipher the underlying mechanism of how pathogens elicit and evade immune responses and the immune homeostasis during infection. Here, we review new findings in Chinese studies concerning immune response to pathogens, immunoregulation and pathogen evasion, which offer us new insight into the trend in infectious immunology in China.

\section{Response to pathogens}

The immune response triggered by microbial pathogens achieves appropriate effector function from diverse pathways to protect the host against destructive invasion. However, most of the underlying mechanism remains unclear.

The first defense employed by the innate immune response is to recognize molecular patterns expressed by invading pathogens via pattern recognition receptors (PRRs). Proteins on pathogens which are involved in driving host immune response are defined. The surface glycoprotein hemagglutinin (HA), the most important protein in molecular epidemiology and pathogenesis of influenza viruses, is characterized in the swine-origin influenza virus $\mathrm{A}$

email: yangw@moon.ibp.ac.cn
(H1N1)-2009 [1]. Tp0751 recombinant protein from T. pallidum is found to induce the production of proinflammatory cytokines and the ensuing immune responses [2]. The PRRs may locate either on the membrane surface e.g. Toll-like receptors (TLRs) or inside the cytoplasm e.g. Nod-like receptors (NLRs). NLRs assemble into multimolecular machines termed inflammasomes to detect intracellular pathogens [3]. Inflammasomes drive inflammatory processes through promoting the maturation of inflammatory cytokines such as interleukin (IL)-1 $\beta$ and IL-18 [4-7]. Alternatively, the recognition of lipopolysaccharide (LPS) is mainly mediated by TLR4/myeloid differentiation protein-2 (MD-2) heterodimers. During this process, residues Glu24-Met41 in the N terminal of TLR4 are involved in TLR4 binding to MD-2 and LPS-stimulated TLR4 aggregation [8]. Signaling pathways initiated by ligand binding to TLRs activate NF- $\kappa \mathrm{B}$, Mitogen-activated protein kinase (MAPK), and interferon (IFN), during in which the tumor necrosis factor (TNF) receptor-associated factor (TRAF) family is shown to participate [9-11]. These responses triggered by PRRs recognizing pathogens culminate activation of antimicrobial killing mechanisms and secretion of cytokines and chemokines. For example, production of broadspectrum antimicrobial peptides is a common innate immunity defense mechanism against infection. As potentially great alternatives to current antibiotics, antimicrobial peptides have been studied in most researches [12-17]. Chen and colleagues have first isolated antibacterial peptides from the ovine reproductive tract [18]. Two novel temporins, one of important families of antimicrobial peptides from Litho- 
bates catesbeianus, have been molecular cloned [19]. The antimicrobial mechanism of these peptides has been investigated. Due to the critical role of type I IFNs in innate antiviral response, their production and downstream signaling cascades are often the hot topics under intensive investigation. Plasmacytoid dendritic cells (pDCs), which sense viral nucleic acids within the endosomal compartments through their TLR7 and TLR9, are professional type I IFN-producing cells. These IFNs not only directly inhibit viral replication but also play an essential role in linking the innate and adaptive immune system [20]. The Mx GTPase pathway is one of the most powerful antiviral mechanisms induced by IFNs. Belonging to the dynamin superfamily of large GTPases, Mx proteins have direct antiviral activity by interfering with viral genome replication [21-23]. Sun and colleagues have well reviewed recent findings in the structural and functional studies of Mx protein, which are significant for prophylaxis and control of the emerging and re-emerging viruses [24].

\section{Immunoregulation}

Although these responses are physiologically designed for the defense against pathogens, their inappropriate or poorly restricted activation drives inflammatory disorders and causes host pathology. Thus, not only pathogen-associated molecular pattern recognition but also inflammatory process are normally under stringent regulation. A novel pathway mediated by CD24 and its receptor is essential in self-nonself discrimination in pattern recognition, enabling to initiate immunity against pathogens without significant immune-mediated self-destruction in case of tissue injuries [25]. Recent studies have revealed that conventional T cells dampen the innate immune response during the early phases of infection, and effector and memory $\mathrm{T}$ cells dampen the hyperactive inflammasome during late phase of primary responses or on secondary challenges, regulating host immunity appropriately [26]. In addition, regulators, such as soluble TLRs, growth arrest-specific gene 6 (Gas6), suppressor of tumorigenicity (ST2) and so on, act at multiple levels within TLR signaling transduction [27,28]. As a transcriptional factor significantly involved in inflammation, Kruppel-like factor 4 (KLF4) inhibits LPS-induced IL-6 release and thus limits potentially harmful immunopathological consequences [29]. Some mechanisms are employed by negative regulators to ensure the appropriate modulation of immune response [30-32].

\section{Pathogen evasion}

In opposition to host protective strategy, some pathogens may be able to evade and subvert immune responses. Programmed death 1 (PD-1), a negative regulator for activation, expansion and acquisition of effector functions of T cells, is well involved in pathogen evasion strategies [33,34]. Although extensive studies have been performed on function of PD-1, as so far regulation mechanism of PD-1 expression has not been reported. Zheng and colleagues have identified and characterized the promoter and upstream regulation region of mouse PD-1, providing important clues for the PD-1 gene transcriptional regulation [35]. In addition, papain-like proteases (PLPs), produced by coronavirus such as SARS and NL63, attenuate the innate response against virus through their IFN antagonism activities. As deubiquitinating enzymes, PLPs block polyI:C-induced activation of Interferon regulatory factor 3 (IRF3) and NF- $\mathrm{BB}$, thus reducing IFN induction. Meanwhile, PLPs deactivate ERIS, an endoplasmic reticulum IFN stimulator, by inhibiting the ERIS dimerization [36-41]. Another mechanism involved in pathogen evasion is induced by IbeT, an E. coli K1 pathogenicity island gene. It has been shown that IbeT contributes to escape from the lysosomes into the cytoplasm for replication after $E$. coli $\mathrm{K} 1$ invasion into human brain microvascular endothelial cells $[42,43]$.

These studies have evaluated immune mechanisms involved in infection in cutting-edged aspects, which will not only help us better understand the process of infection but also lay a foundation for developing novel therapies for infectious diseases.

1 Sun Y P, Shi Y, Zhang W, et al. In silico characterization of the functional and structural modules of the hemagglutinin protein from the swine-origin influenza virus A (H1N1)-2009. Sci China Life Sci, 2010, 53: 633-642

2 Liu S Q, Wang S P, Wu Y M, et al. Production of proinflammatory cytokines in the human THP-1 monocyte cell line following induction by Tp0751, a recombinant protein of Treponema pallidum. Sci China Life Sci, 2010, 53: 229-233

$3 \mathrm{Wu} \mathrm{S}$, Metcalf J P, Wu W. Innate immune response to influenza virus. Curr Opin Infect Dis, 2011, 24: 235-240

4 Zhu X M, Yao Y M, Sheng Z Y. Inflammasome and inflammatory response. Prog Biochem Biophys, 2010, 37: 129-137

5 Gong Y N, Wang X, Wang J, et al. Chemical probing reveals insights into the signaling mechanism of inflammasome activation. Cell Res, 2010, 20: 1289-1305

6 Li H, Fang X M. Research advances of inflammasome and its role in sepsis. J Zhejiang Univ (Med Sci), 2010, 39: 487-492

7 Chen M, Wang H, Chen W, et al. Regulation of adaptive immunity by the NLRP3 inflammasome. Int Immunopharmacol, 2011, 11: 549-554

8 Zhong T Y, Tang J, Chen D Y, et al. Using FRET to study the interaction domain of TLR4 binding to MD-2 in living cells. Prog Biochem Biophys, 2009, 36: 1451-1457

9 Yang Y X, Li G Y. Progression of lipopolysaccharide signal pathway. J Cent South Univ(Med Sci), 2006, 31: 141-145

10 Duan X Z, Wang H F. The role of LPS/TLR4 interaction on the pathogenesis of acute on chronic liver failure. Chinese J Hepatol, 2010, 18: 78-80

11 Wang YY, Zhang P, Liu Y F, et al. TRAF-mediated regulation of immune and inflammatory responses. Sci China Life Sci, 2010, 53: $159-168$

$12 \mathrm{Li} \mathrm{M}, \mathrm{Yu} \mathrm{D} \mathrm{H}$, Cai $\mathrm{H}$. The synthetic antimicrobial peptide KLKL5KLK enhances the protection and efficacy of the combined DNA vaccine against Mycobacterium tuberculosis. DNA Cell Biol, 
2008, 27: 405-413

13 Hao G, Shi Y H, Tang Y L, et al. The membrane action mechanism of analogs of the antimicrobial peptide Buforin 2. Peptides, 2009, 30: 1421-1427

14 Zhang X G, Fang C, Bai H, et al. Research progress on mechanism of antimicrobial peptides. Prog Physiol sci, 2011, 42: 11-15

15 Dang X L, Wang Y S, Huang Y D, et al. Purification and characterization of an antimicrobial peptide, insect defensin, from immunized house fly (Diptera: Muscidae). J Med Entomol, 2010, 47: 1141-1145

16 Wang K J, Cai J J, Cai L, et al. Cloning and expression of a hepcidin gene from a marine fish (Pseudosciaena crocea) and the antimicrobial activity of its synthetic peptide. Peptides, 2009, 30: 638-646

17 Wang Y, Lu Z, Feng F, et al. Molecular cloning and characterization of novel cathelicidin-derived myeloid antimicrobial peptide from Phasianus colchicus. Dev Comp Immunol, 2011, 35: 314-322

18 Chen C, Wang X H, Bo X W. Antibacterial peptides of the ovine reproductive tract. Prog Biochem Biophys, 2009, 36: 1483-1489

19 Zhao R L, Han J Y, Han W Y, et al. Molecular cloning of two novel temporins from Lithobates catesbeianus and studying of their antimicrobial mechanisms. Prog Biochem Biophys, 2009, 36: 1064-1070

20 Tang F, Du Q, Liu Y J. Plasmacytoid dendritic cells in antiviral immunity and autoimmunity. Sci China Life Sci, 2010, 53: 172-182

21 Li G, Zhang J, Sun Y, et al. The evolutionarily dynamic IFN-inducible GTPase proteins play conserved immune functions in vertebrates and cephalochordates. Mol Biol Evol, 2009, 26: 1619-1630

22 Haller O, Stertz S, Kochs G. The Mx GTPase family of interferon-induced antiviral proteins. Microbes Infect, 2007, 9: 1636-1643

23 Haller O, Staeheli P, Kochs G. Interferon-induced Mx proteins in antiviral host defense. Biochimie, 2007, 89: 812-818

24 Sun Y P, Gao George F. Structural and functional studies of the innate immune effector Mx proteins: a review. Prog Biochem Biophys, 2010, 37: 699-706

25 Liu Y, Chen G Y, Zhang P, et al. On self-nonself discrimination in pattern recognition. Sci China Life Sci, 2010, 53: 169-171

26 Tang $\mathrm{H}, \mathrm{Fu} \mathrm{Y}$ X. A new role for $\mathrm{T}$ cells in dampening innate inflammatory responses. Sci China Life Sci, 2010, 53: 190-194

27 Sun B, Han D S. Negative regulation of TLR signaling pathways. Prog Biochem Biophys, 2009, 36: 1516-1522

28 Wang J, Hu Y, Deng W W, et al. Negative regulation of Toll-like receptor signaling pathway. Microbes Infect, 2009, 11: 321-327

29 Feng Y S, Liu M D, Liu Y, et al. Role of KLF4 in Regulating the Expression of IL-6 Induced by LPS. Prog Biochem Biophys, 2009, 36: 1313-1318

30 Hou J, Wang P, Lin L, et al. MicroRNA-146a feedback inhibits RIG-I-dependent Type I IFN production in macrophages by targeting
TRAF6, IRAK1, and IRAK2. J Immunol, 2009, 183: 2150-2158

31 Sun B, Qi N, Shang T, et al. Sertoli cell-initiated testicular innate immune response through toll-like receptor-3 activation is negatively regulated by Tyro3, Axl, and mer receptors. Endocrinology, 2010, 151: 2886-2897

32 Seit-Nebi A, Cheng W, Xu H, et al. MLK4 has negative effect on TLR4 signaling. Cell Mol Immunol, 2011, doi:10.1038/cmi.2011.15.

33 Xie Z, Chen Y, Zhao S, et al. Intrahepatic PD-1/PD-L1 up-regulation closely correlates with inflammation and virus replication in patients with chronic HBV infection. Immunol Invest, 2009, 38: 624-638

34 Wu Y Y, Lin C W, Cheng K S, et al. Increased programmed death-ligand-1 expression in human gastric epithelial cells in Helicobacter pylori infection. Clin Exp Immunol, 2010, 161: 551-559

35 Zheng H Y, Tang B K, Wang P W, et al. Identification and characterization of the promoter and upstream regulation region of mouse PD-1. Prog Biochem Biophys, 2010, 37: 527-533

36 Sun L, Liu D B, Yang Y D, et al. Regulation of antiviral innate immune responses by human coronavirus. Prog Biochem Biophys, 2010, 37: 239-244

37 Sun L, Yang Y D, Liu D B, et al. Deubiquitinase activity and regulation of antiviral innate immune responses by papain-like proteases of human coronavirus NL63. Prog Biochem Biophys, 2010, 37: 871880

38 Wang G, Chen G, Zheng D, et al. PLP2 of mouse hepatitis virus A59 (MHV-A59) targets TBK1 to negatively regulate cellular type I interferon signaling pathway. PLoS One, 2011, 6: e17192

39 Wang D, Fang L, Li P, et al. The leader proteinase of foot-and-mouth disease virus negatively regulates the type I interferon pathway by acting as a viral deubiquitinase. J Virol, 2011, 85: 3758-3766

40 Li S W, Lai C C, Ping J F, et al. Severe acute respiratory syndrome coronavirus papain-like protease suppressed alpha interferon-induced responses through downregulation of extracellular signal-regulated kinase 1-mediated signalling pathways. J Gen Virol, 2011, 92: 11271140

41 Zheng D, Chen G, Guo B, et al. PLP2, a potent deubiquitinase from murine hepatitis virus, strongly inhibits cellular type I interferon production. Cell Res, 2008, 18: 1105-1113

42 Zhang K, Zhao W D, Li Q, et al. IbeT, a Escherichia coli K1 pathogenicity island gene, is essential for escape from the lysosomes in human brain microvascular endothelial cells. Prog Biochem Biophys, 2009, 36: 417-423

43 Zou Y, He L, Chi F, et al. Involvement of Escherichia coli K1 ibeT in bacterial adhesion that is associated with the entry into human brain microvascular endothelial cells. Med Microbiol Immunol, 2008, 197: $337-344$

Open Access This article is distributed under the terms of the Creative Commons Attribution License which permits any use, distribution, and reproduction in any medium, provided the original author(s) and source are credited. 